\title{
A novel cell type-specific role of $p 38 \alpha$ in the control of autophagy and cell death in colorectal cancer cells
}

\author{
F Comes ${ }^{1,6}$, A Matrone ${ }^{1,6}$, P Lastella ${ }^{1}$, B Nico ${ }^{2}$, FC Susca ${ }^{1}$, R Bagnulo ${ }^{1}$, G Ingravallo ${ }^{3}$, S Modica ${ }^{4}$, G Lo Sasso ${ }^{4}$, A Moschetta ${ }^{4}$, \\ G Guanti* ${ }^{\star, 1}$ and C Simone ${ }^{\star, 1,5}$
}

Cancer develops when molecular pathways that control the fine balance between proliferation, differentiation, autophagy and cell death undergo genetic deregulation. The prospects for further substantial advances in the management of colorectal cancer reside in a systematic genetic and functional dissection of these pathways in tumor cells. In an effort to evaluate the impact of p38 signaling on colorectal cancer cell fate, we treated HT29, Caco2, Hct116, LS174T and SW480 cell lines with the inhibitor SB202190 specific for p38 $\alpha / \beta$ kinases. We report that p38 $\alpha$ is required for colorectal cancer cell homeostasis as the inhibition of its kinase function by pharmacological blockade or genetic inactivation causes cell cycle arrest, autophagy and cell death in a cell type-specific manner. Deficiency of $\mathrm{p} 38 \alpha$ activity induces a tissue-restricted upregulation of the GABARAP gene, an essential component of autophagic vacuoles and autophagosomes, whereas simultaneous inhibition of autophagy significantly increases cell death by triggering apoptosis. These data identify p38 $\alpha$ as a central mediator of colorectal cancer cell homeostasis and establish a rationale for the evaluation of the pharmacological manipulation of the p38 $\alpha$ pathway in the treatment of colorectal cancer.

Cell Death and Differentiation (2007) 14, 693-702. doi:10.1038/sj.cdd.4402076; published online 8 December 2006

Colorectal cancer is a major health concern, with more than 1000000 new cases and 500000 deaths expected worldwide per year. ${ }^{1}$ Prognostic evaluation is currently based on histological appearance, and there are no molecular markers internationally recognized as standard predictor factors. The conventional therapy involving surgery and adjuvant therapy seems to give rise to improvements in progression-free and overall survival. Nevertheless about $50 \%$ of patients die within 5 years owing to metastasis or recurrent disease. ${ }^{2}$ The prospects for further substantial advances in the management of colorectal cancer reside in a systematic genetic and functional dissection of cell cycle and cell death regulatory pathways in tumor cells in order to identify differential cellular effects of agents that may have a direct impact on cancer therapy.

During the last decade, a number of deacetylase inhibitors (DI) have been identified. These DI induce tumor cells to undergo growth arrest, differentiation, and/or apoptosis in culture and in animal models, at doses that seem to be nontoxic and appear to be selective. Butyrate, a DI that is naturally formed in the human colon, is able to reduce the size and the number of tumors in rat models of bowel cancer. ${ }^{3}$ In vitro, sodium butyrate $(\mathrm{NaB})$ is a potent differentiating agent for several colorectal cancer cell lines (CRCs). ${ }^{4-6} \mathrm{NaB}-$ mediated cell cycle withdrawal of CRCs appears to be dependent on acetylation of histones and consequent changes in transcription, requiring continuous protein synthesis and the expression of $21 .^{7}$ It has recently been shown that $1 \mathrm{mM} \mathrm{NaB}$ is the best working concentration to activate the differentiation program in CRCs without triggering apoptosis, whereas $5 \mathrm{mM}$ $\mathrm{NaB}$ is sufficient to induce a p53-independent apoptotic cell death by activating a pathway involving p38, PPAR $\gamma$ and caspases. $^{6,8}$

Several intracellular signaling cascades have been implicated in the regulation of the proliferation-differentiation balance in enterocytes by the coordinated expression of the genome in response to environmental cues. During enterocyte differentiation of CRCs the ERK pathway is switched off, whereas $\mathrm{Pl}_{3} \mathrm{~K}$ class $\mathrm{I}$ and $\mathrm{p} 38$ activities are increased and essential for full differentiation. ${ }^{5,9}$ It has been suggested that p38 $\alpha$ could activate the intestinal-specific homeobox transcription factor CDX2 probably by phosphorylation. ${ }^{10}$ This mechanism resembles that described for $p 38 \alpha / \beta$ in muscle cells, where they phosphorylate the transcription factor MEF2 and the SWI/SNF subunit BAF60 to remodel chromatin and activate tissue-specific transcription. ${ }^{11,12}$ Interestingly, chromatin remodeling complex dysfunction has been described in human colorectal cancer cells. ${ }^{13}$

The p38 pathway is often activated by stressful stimuli and cytokines leading to diverse cell type-specific responses, such

\footnotetext{
${ }^{1}$ Division of Medical Genetics, Department of Biomedicine in Childhood, University of Bari, Bari, Italy; ${ }^{2}$ Department of Human Anatomy and Histology, University of Bari, Bari, Italy; ${ }^{3}$ Department of Pathological Anatomy, University of Bari, Bari, Italy; ${ }^{4}$ Consorzio Mario Negri Sud, Santa Maria Imbaro (Ch) and 'Clinica Medica Murri', Department of Internal Medicine, University of Bari, Bari, Italy and ${ }^{5}$ Sbarro Institute for Cancer Research and Molecular Medicine, Center for Biotechnology, College of Science and Technology, Temple University, Philadelphia, USA

*Corresponding authors: G Guanti or C Simone, Division of Medical Genetics, Department of Biomedicine in Childhood, University of Bari, Bari, Italy.

Fax: + 390805478269; E-mails: guanti@medgene.uniba.it or csimone@temple.edu

${ }^{6}$ These two authors contributed equally to this work.

Keywords: colorectal cancer; p38 $\alpha$; autophagic cell death; apoptosis; SB202190

Abbreviations: DI, deacetylase inhibitors; NaB, sodium butyrate; CRCs, colorectal cancer cell lines; SI, sucrose isomaltase; SB, SB202190; PAS, periodic-acid Schiff; IL, intracellular lumens; IC, intercellular cysts; 3MA, 3-methyladenine; hFSF, human fetal skin fibroblasts

Received 28.7.06; revised 24.10.06; accepted 31.10.06; Edited by Y Tsujimoto; published online 08.12 .06
} 
as cell survival or apoptosis. In non-transformed cells, p38 $\alpha$ is required for inhibition of proliferation induced by cell-to-cell contact, as happens in primary cultures of human fibroblasts. ${ }^{14}$ In cancer cells, p38 $\alpha / \beta$ are required for breast and colorectal cancer cell migration and metastasis formation in mice. ${ }^{15,16}$ However, over-activation of p38 by a constitutively active form of its upstream kinase (MKK6EE) eliminates the tumor-prone phenotype of mice obtained by intercrossing transgenic mice with breast-restricted expression of Wip1, the p53-induced phosphatase targeting p38 and the oncogene ErbB2. ${ }^{17}$ Moreover, $\mathrm{p} 38 \alpha / \beta$ contribute to TGF $\beta$-dependent tumor progression by inducing epithelial to mesenchymal transdifferentiation and cell migration, ${ }^{18}$ and to rat chondrosarcoma cell proliferation. ${ }^{19}$

In an effort to elucidate the impact of p38 in the molecular mechanisms regulating $\mathrm{CRC}$ fate, we analyzed gene expression profile of HT29 cells growing logarithmically, confluent in culture and induced to differentiate. Five genes were considered, three of them were cell cycle-related genes: cyclin $E$ is expressed during the late $G 1$ phase of the cell cycle until the end of the S-phase, cyclin A starts to accumulate during $S$ phase and is abruptly destroyed before metaphase, and, finally, p21 is involved in the control of G1/S and G2/M transitions. The other two genes codify for tissue-specific proteins: sucrose isomaltase ( $\mathrm{SI})$ is an intestinal disaccharidase expressed in the brush border of mature enterocytes; villin is a cytoskeletal protein specific for microvilli.

At the onset of differentiation, the pharmacological blockade of the p38 cascade inhibits the expression of p21, SI and villin confirming the role of this pathway in the differentiation program of distinct cell types. ${ }^{9-12}$ Surprisingly, we discovered that $p 38 \alpha$ is required for $\mathrm{CRC}$ proliferation and survival as pharmacological blockade or genetic depletion of its kinase activity induced growth arrest, autophagy and cell death in a cell type-specific manner. Interestingly, the inhibition of the autophagic activity of these cells induces a dramatic increase in cell death by triggering apoptosis.

\section{Results}

Pharmacological blockade of p38 affects proliferation and differentiation of HT29 cells. To characterize the molecular mechanisms involving the p38 pathway in the control of colorectal cancer cell fate, we employed the human HT29 cell line that recapitulates large bowel cell differentiation in vitro. The effect of p38 inhibition by the specific compound SB202190 (SB) was evaluated by analyzing the expression of cyclin E, cyclin A, p21, SI and villin transcripts in HT29 cells growing logarithmically, confluent in culture or induced to differentiate with $\mathrm{NaB}$ $1 \mathrm{mM}$ for $96 \mathrm{~h}$ (Figure 1a). SB addition to logarithmically growing cultures caused a significant reduction of cyclin $\mathrm{E}$ expression already within the first $24 \mathrm{~h}$, whereas it downregulated cyclin A transcription only after $72 \mathrm{~h}$ of treatment (Figure 1b). Moreover, despite the significant reduction in the proliferation rate, the levels of p21 were consistently lower than those of untreated cells (Figure 1b). Conversely, in confluent cultures, SB only slightly affected p21 expression induced by cell-to-cell contact (Figure 1b). In differentiating HT29 cells, p38 blockade counteracted the effect of $\mathrm{NaB}$ on the silencing of cyclins, induction of p21 and expression of enterocyte-specific markers (Figure 1b). Collectively these data suggested that the p38 pathway is involved in the transcription control of both cell cycle and tissue-specific genes.

p38 blockade induces autophagic cell death in HT29 cells. Very surprisingly, p38 blockade in HT29 cells induced the formation of large cytoplasmic vacuoles regardless of culture conditions (Figure 2), whereas PD98059 and LY294002, two inhibitors specific for MEK1 and $\mathrm{PI}_{3} \mathrm{~K}$ kinases, respectively, did not (data not shown). They appeared approximately $2 \mathrm{~h}$ after SB addition, then became larger reaching the maximum diameter at $24 \mathrm{~h}$ $(19.3 \pm 10.6 \mu \mathrm{M})$ (Figure 2a). The percentage of cells presenting vacuoles was time dependent as well. At $24 \mathrm{~h}$ more than $90 \%$ of cells presented at least one vacuole filling up most of the cytoplasmic compartment (Figures 2 and $4 a$ ).

The observation that SB treatment caused growth inhibition and the formation of intracellular vacuoles, prompted us to investigate their nature. To this end we employed different cytological methods such as periodic-acid Schiff (PAS), Papanicolaou, Sudan black B and Oil red O. Among them only PAS demonstrated a dot-like staining within vacuoles suggesting the presence of glycoproteins (Figure 2b). Electron microscopy showed that SB-treated cells were roundly shaped and displayed a dark cytoplasm containing a heterochromatic nucleus. Numerous large vacuoles filled up with dense material, autophagic vacuoles containing cell organelle debris and double-membrane autophagosomes were recognized (Figure 3). In untreated HT29 cells no molecular features of autophagy were detected (data not shown). Quite different was the morphology of fully differentiated HT29 cells, which appeared elongated, and displayed euchromatic nuclei and electron clear cytoplasms with numerous mitochondria and a few small empty vacuoles. Moreover, circular structures coated with regular microvilli, often located at the apical cellular surface (intracellular lumens, IL) or between the cells (intercellular cysts, IC), were observed (Figure 3). In HT29 cells either untreated or treated with SB no IL or IC were detected (Figure 3 and data not shown).

The detection of these ultrastructural findings, defined as the 'golden standard' for assessing autophagic activity, ${ }^{20}$ clearly indicated that pharmacological blockade of p38 induced an autophagic response in HT29 cells. Further evidence was provided by the pretreatment with 3-methyladenine (3MA) or with the inhibitor of translation puromycin, two well-known inhibitors of the autophagic process, that severely impaired SB-dependent induction of autophagy in HT29 cells (Figure 4a). To better characterize this process, we evaluated the expression of the GABARAP gene, one of the human homologs of the yeast ATG8 gene. Atg8 is a member of a novel ubiquitin-like protein family and an essential component of the autophagic machinery. In yeast, it is upregulated by rapamycin-dependent Tor inhibition and nitrogen starvation. ${ }^{21}$ In HT29 cells, GABARAP expression levels were significantly augmented by p38 blockade and correlated with autophagic vacuoles and autophagosome 
a

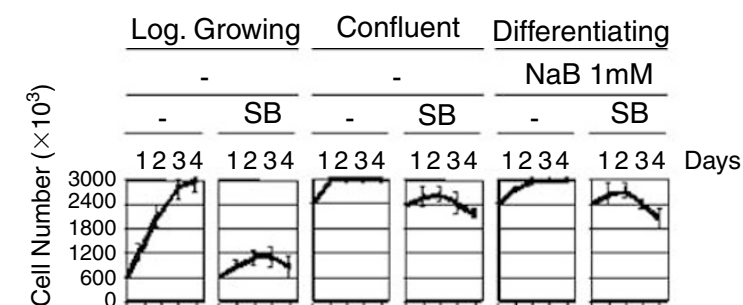

b

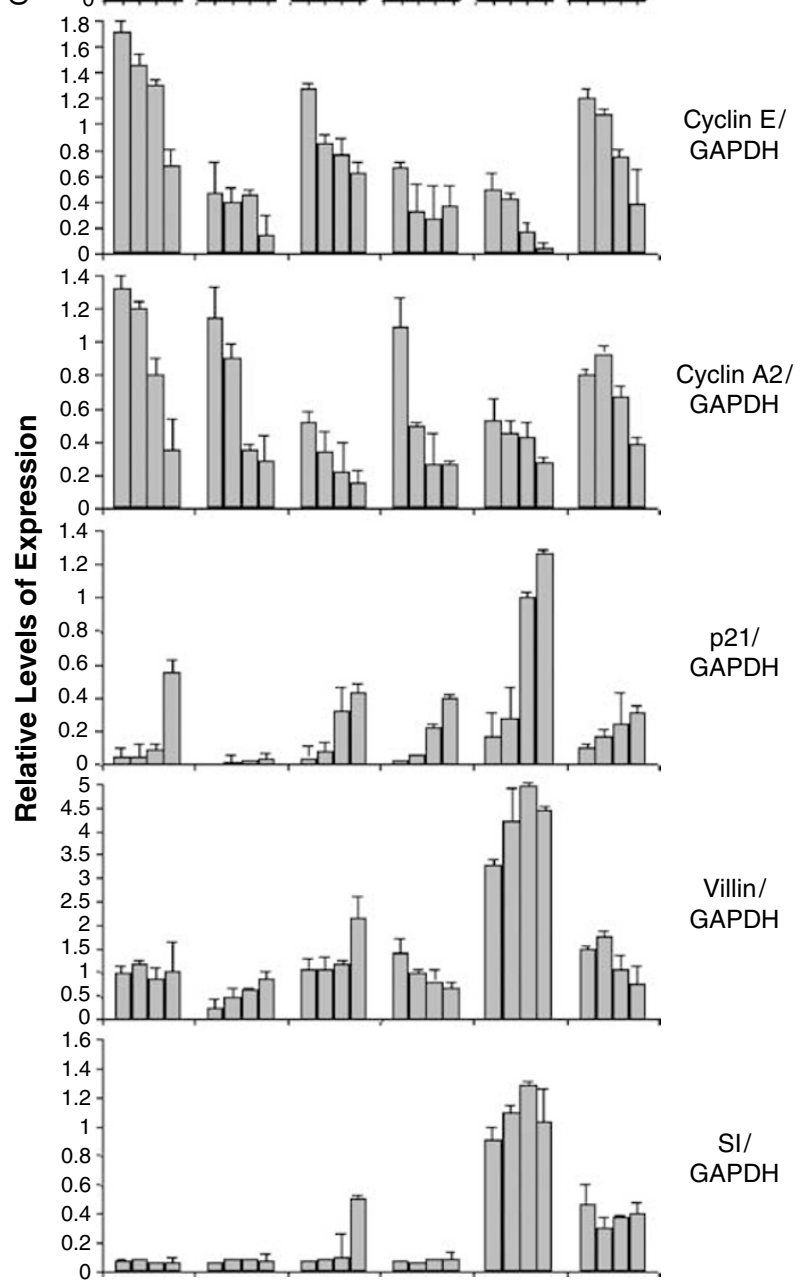

C

Relative PCR Levels

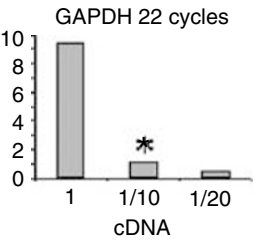

Cyclin E 28 cycles

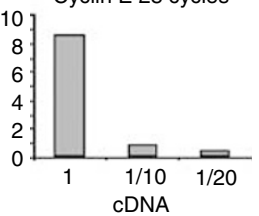

Cyclin A 28 cycles

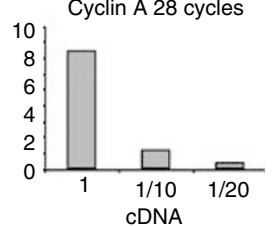

p21 28 cycles

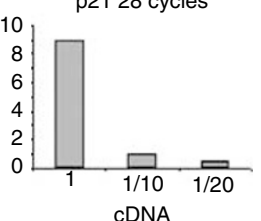

Villin 28 cycles

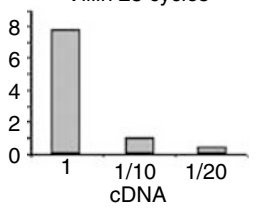

SI 27 cycles

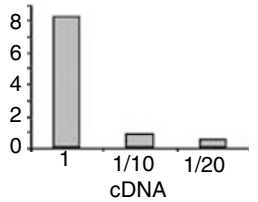

Figure 1 Inhibition of p38 by SB202190 modifies the expression profile of HT29 cells growing logarithmically, confluent in culture and induced to differentiate. (a) Cells were plated at different density in either the absence or presence of NaB and SB202190 (SB) and cultured for up to $96 \mathrm{~h}$. (b) Relative levels of expression of the indicated genes were calculated by GeneScan and normalized on GAPDH signal (see Supplementary Figure 1). (c) For each set of primers a standard curve was constructed using serial dilutions of CDNA to define the dynamic range and calculate the amplification efficiency. Relative PCR levels were estimated by normalization on the GAPDH fluorescent signal obtained by the amplification of 1/10 cDNA dilution (see asterisk)

formation (Figure 4a and b), whereas rapamycin failed to induce both autophagy and GABARAP upregulation in these cells (data not shown). Interestingly, 3MA failed to impair the SB-mediated induction of GABARAP expression (Figure 4b). This is noteworthy as 3MA prevents autophagy at an early stage by inhibiting $\mathrm{PI}_{3} \mathrm{~K}$ class III, which interacts with Atg6/ Beclin- 1 to promote the nucleation of autophagic vesicles. ${ }^{22,23}$ Our data suggest that the p38 control of GABARAP transcription is independent from this first step.
In addition to the above-mentioned growth suppression of HT29 cells (Figure 1a), SB exposure significantly reduced the number of viable cells compared to the untreated control cells (relative viability) by triggering cell death (Figure $4 c$ and $d$ ). Non-viable SB-treated cells showed the characteristic features of necrotic-like cells. In fact, they took up ethidium bromide, a marker of plasma membrane disruption, but maintained the nucleus intact until late stages, and showed only a partial chromatin condensation without a significant 


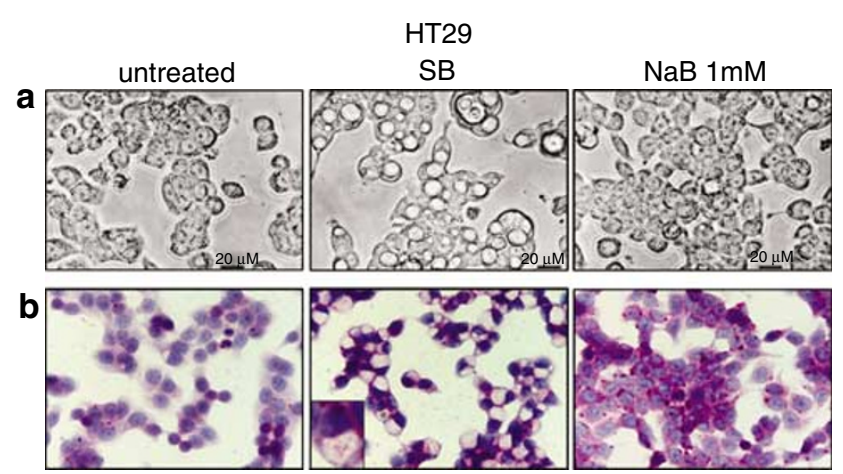

Figure 2 p38 blockade induces the formation of macrovacuoles in HT29 cells. HT29 cells were cultured in either the absence or presence of $\mathrm{NaB} 1 \mathrm{mM}$ or SB202190 (SB) for $24 \mathrm{~h}$. (a) Living cells observed and photographed with a phasecontrast microscope. (b) Cells fixed and stained with PAS showing nucleolated nuclei and granular cytoplasms (untreated); hyperchromatic nuclei and single intracytoplasmic macrovacuole (SB); nucleolated nuclei and granular cytoplasms with PAS positive deposits (NaB). SB-induced macrovacuoles show a dot-like staining (inset). Original magnification $\times 400$

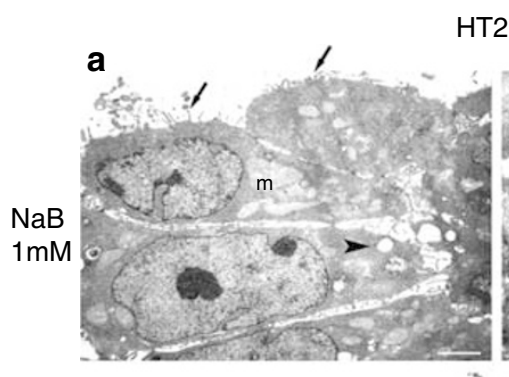

HT29

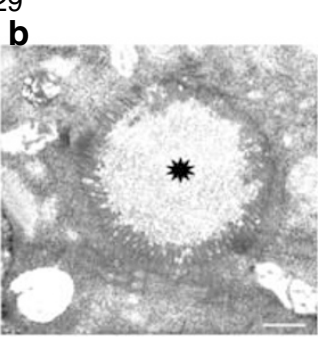

C

?.

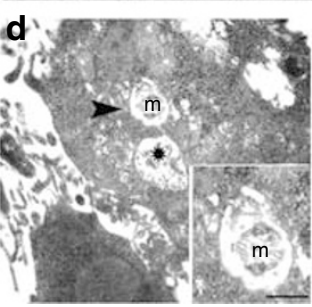

Figure 3 Electron microscopy of $\mathrm{HT} 29$ cells cultured in the presence of $\mathrm{NaB}$ $1 \mathrm{mM}(96 \mathrm{~h}$ ) or SB202190 (SB; $24 \mathrm{~h}$ ). (a) NaB-treated cells showing euchromatic nuclei and cytoplasms containing a few vacuoles (arrowhead) and numerous mitochondria $(\mathrm{m})$. Microvilli (arrow) are present on the external plasma membrane. (b) An IC coated by regular microvilli (asterisk). (c, d) SB-treated cells show heterochromatic nuclei (n) and dark cytoplasms filled up with large vacuoles containing dense material (asterisk), autophagic vacuoles containing debris of a mitochondrium $(\mathrm{m})$ (d, inset), double-membrane autophagosomes (c, white arrow) and lipidic droplets (c, arrow). Scale bar: (a and c) $0.7 \mu \mathrm{m}$; (b) $0.4 \mu \mathrm{m}$; (d) $0.57 \mu \mathrm{m}$

increase in DNA fragmentation (Figure $4 \mathrm{e}-\mathrm{g}$ ). The coexistence of autophagy with features of non-apoptotic death is considered as an hallmark of autophagic cell death or type II programmed cell death. ${ }^{20,24}$ Notably, pharmacological blockade of SB-dependent autophagy not only failed to increase viability, but it even increased cell death by inducing a switch from autophagic cell death to apoptosis. In fact, cells pretreated with $3 \mathrm{MA}$ and then exposed to SB showed cell shrinkage, severe chromatin condensation, nuclear fragmen- tation and DNA degradation comparable to cells exposed to $\mathrm{NaB} 5 \mathrm{mM}$ (Figure 4e-g).

SB-dependent induction of autophagy relies only upon p38 $\alpha$ blockade. As SB specifically inhibits p38 $\alpha$ and $\beta$ gene products, we investigated whether both isoforms were synthesized in HT29 cells. It was previously reported that Caco2 cells exhibit distinct profiles of p38 isoform expression depending on the differentiation status. Undifferentiated cells produce $\mathrm{p} 38 \alpha, \beta$ and $\gamma$, whereas differentiated cells synthesize p38 $\alpha, \gamma$ and $\delta .{ }^{25}$ Conversely, the expression profile of p38 isoforms in HT29 cells remained unchanged regardless of the differentiation status. In fact, the cells expressed $\mathrm{p} 38 \alpha, \gamma$ and $\delta$ despite $\mathrm{NaB}$ treatment (Figure $5 \mathrm{a}$ and $b$ ). The absence of $\mathrm{p} 38 \beta$ transcripts suggested that SB-mediated autophagic response relies only upon $p 38 \alpha$ blockade. To test this hypothesis, we used siRNA gene silencing targeting $\mathrm{p} 38 \alpha$ and $\beta$ gene in HT29 cells (Figure $5 \mathrm{e}$ ). The siRNA against $\mathrm{p} 38 \alpha$ reduced the target mRNA and protein levels by $70-80 \%$, whereas the siRNA against the $\beta$ isoform did not affect nor p38 $\alpha$ mRNA neither total $\mathrm{p} 38$ protein expression in accordance with the absence of $\mathrm{p} 38 \beta$ in these cells (Figure $5 \mathrm{c}$ ). Evaluation of the autophagic activity and GABARAP expression in siRNAtransfected cells clearly indicated that transient genetic ablation of p38 $\alpha$ was able to induce autophagy in HT29 colorectal cancer cells (Figure $5 \mathrm{~d}$ ).

Colorectal cancer-specific induction of autophagic cell death by $p 38 \alpha$ inhibition. To get further insight into the involvement of $p 38 \alpha$ in the autophagic response, we extended our investigation to Caco2, SW480, LS174T and Hct116 CRCs (Tables 1 and 2). Cytological and morphological evaluation confirmed the SB-mediated induction of growth arrest and autophagic cell death in all CRC lines (Figures 6ac). Consistently, SB-mediated upregulation of GABARAP was detected in all cell lines tested (Figure 6e). Pharmacological blockade of autophagy drastically increased cell death by promoting a switch from autophagic death to apoptosis (Figure 6c, and data not shown).

To investigate whether p38 $\alpha$ inhibition induced type II cell death in a cell type-specific manner, Hep3B liver carcinoma cells, HeLa cervical carcinoma cells, HEK293 embryonic kidney cells, and human fetal skin fibroblasts (hFSF) were exposed to SB for $24 \mathrm{~h}$ (Figure $6 \mathrm{~d}$ and Table 2). Pharmacological inhibition of p38 failed to induce cell cycle arrest, autophagy and cell death, and it did not promote GABARAP upregulation in any of the cell lines tested (Figure $6 \mathrm{~d}$ and $\mathrm{e}$, and data not shown).

The analysis of the relative abundance of $\mathrm{p} 38 \alpha$ and $\beta$ transcripts in all cell lines studied, suggested that the cell typespecific effect caused by SB is independent of the expression of $\mathrm{p} 38 \beta$ and relies only upon the presence of $\mathrm{p} 38 \alpha$ in CRCs (Figure 6f).

Moreover, SB treatment failed to cause autophagic cell death in $\mathrm{C} 3 \mathrm{H} 10 \mathrm{~T} 1 / 2$ and NIH3T3 mouse fibroblasts, C2C12 mouse myoblasts and human RD rhabdomyosarcoma cells (Table 2). All together, these data suggest that $p 38 \alpha$ blockade induces growth inhibition, autophagy and cell death in CRCs in a cell type-specific manner. 
a

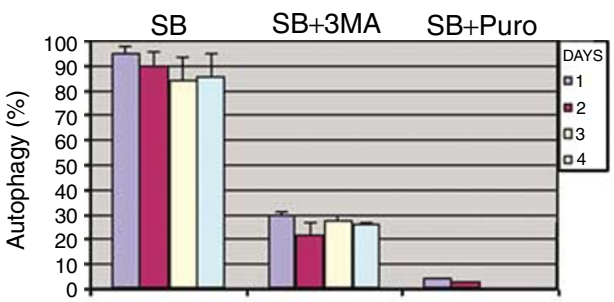

C

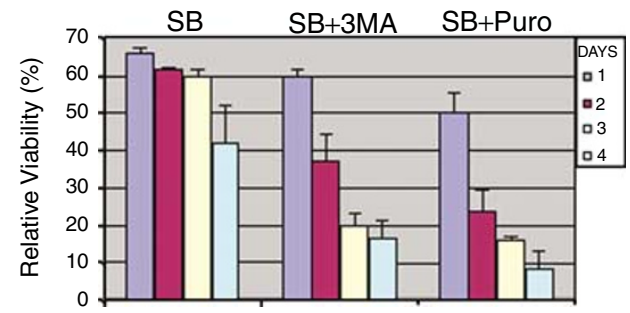

e

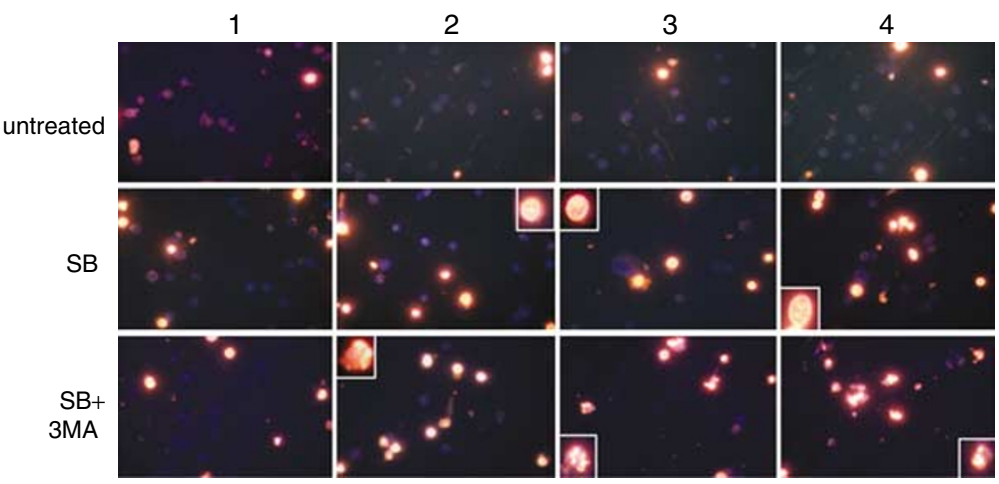

b

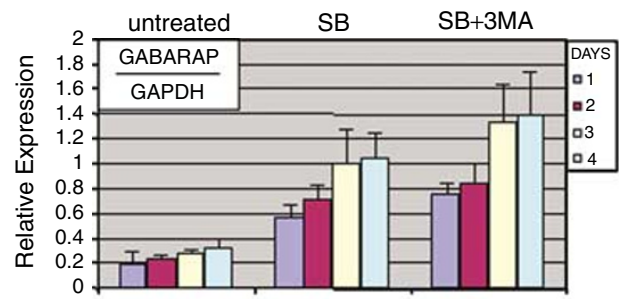

d

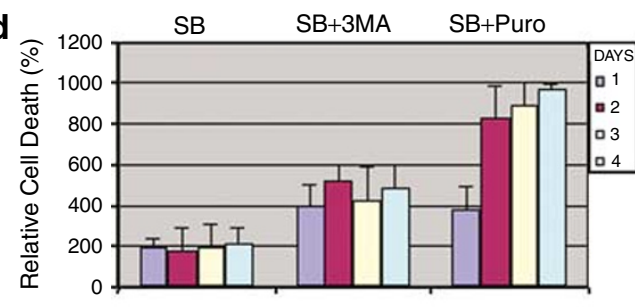

$\mathbf{f}$

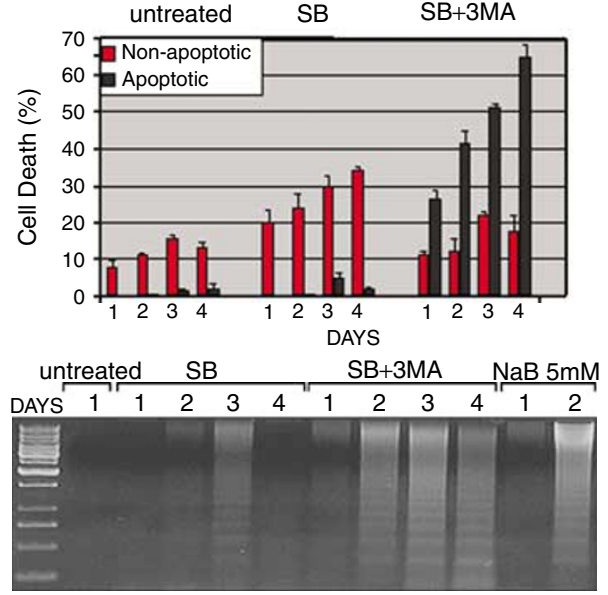

Figure 4 p38 blockade induces autophagic cell death in HT29 cells, whereas inhibition of SB202190-dependent autophagy triggers apoptosis. HT29 cells were cultured in the absence or presence of SB202190 (SB) or a combination of SB202190 and 3-methyiladenine (3MA) or SB202190 and puromycin (Puro) for the indicated periods of time. (a) The percentage of autophagic cells was estimated by phase-contrast microscope and then verified by electron microscope evaluation. (b) Time course of GABARAP gene expression. Relative levels of GABARAP expression were measured by GeneScan evaluation of fluorescent amplifications and normalized on GAPDH signals. (c-f) HT29 cells were collected at the indicated periods of time and mixed with trypan blue solution or a dye mix containing DAPI and ethidium bromide. (c) Relative viability (\%) was estimated by calculating the ratio between viable cells in treated (SB, SB + 3MA, SB + Puro) versus untreated (-) HT29. (d) Relative cell death (\%) represents the ratio between dead cells in treated versus untreated HT29. (e) HT29 cells stained with DAPI + ethidium bromide and analyzed by fluorescent microscope $(\times 400)$. (f) The ratio between apoptotic and necrotic dead cells was calculated and reported as a percentage of the total number of cell counted (g) Genomic DNA was extracted from HT29 cells and loaded into a $1 \%$ agarose gel

\section{Discussion}

We demonstrated that $p 38 \alpha$ is one of the essential kinases for colorectal cancer cells fate and provided evidences indicating that $p 38 \alpha$ blockade causes growth arrest and autophagic cell death. In CRCs, p38 $\alpha$ is required for both cell cycle progression and NaB-dependent induction of cell differentiation. Furthermore, our experiments reveal that $p 38 \alpha$ is required for survival of CRCs growing logarithmically, confluent in culture or induced to differentiate. In fact, p38 blockade induces an autophagic response in these cells associated to growth suppression and cell death in a p53- and p21-independent manner.

The involvement of p38 activity in the negative control of autophagy has been evoked in human liver response to cellular hydration state, but not in mouse myotube response to amino-acid starvation. ${ }^{26}$ In yeasts,
Hog1, the homolog of p38, plays a role in the stabilization machinery of nitrogen deprivation-induced autophagy during ambient osmolarity changes. ${ }^{27}$ In logarithmically growing CRCs, p38 $\alpha$ inhibition induces a cell typespecific upregulation of the human homolog of Atg8 (GABARAP), suggesting that $p 38 \alpha$ is required to exert a negative control on the expression of this gene. In yeasts, the Atg8 gene is upregulated by rapamycin-dependent Tor inhibition, and its protein product is an essential component of the autophagic vescicle formation/size expansion machinery. ${ }^{21}$ In CRCs, rapamycin failed to cause both autophagy and upregulation of GABARAP expression. To date, three Atg8 homologs have been identified in mammalian cells: GATE-16, GABARAP and MAP1LC3. All three proteins appear to be modified with lipids in the same manner described in yeasts, and have been shown to localize to the autophagosome. ${ }^{28}$ 
a

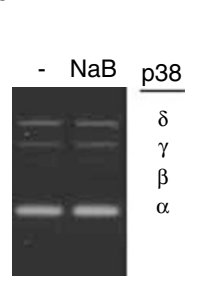

Multiplex PCR b

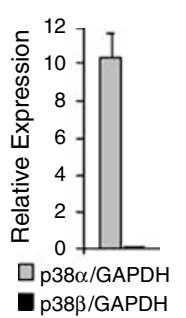

C

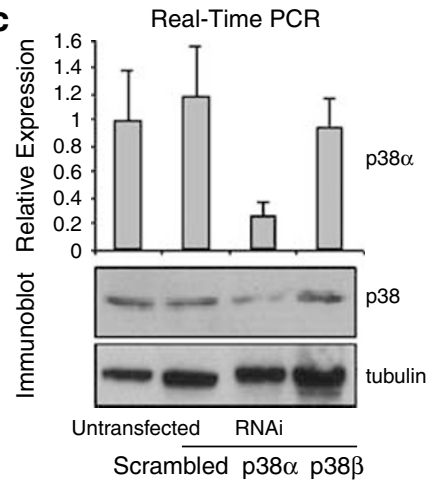

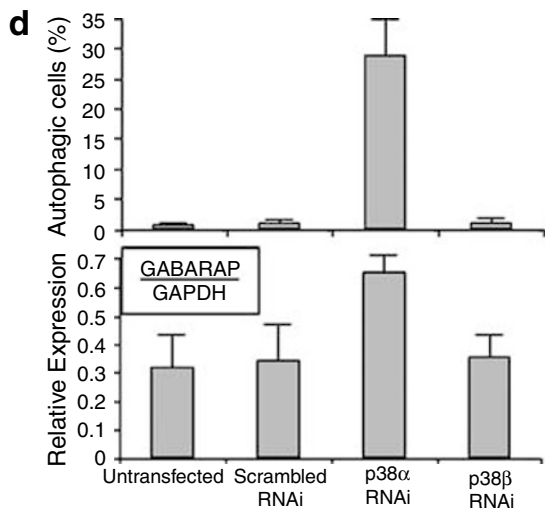

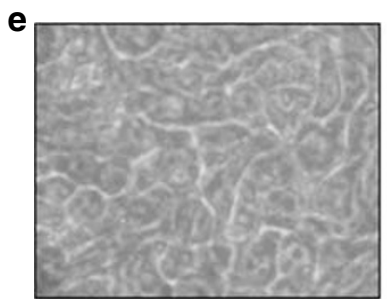

Untransfected

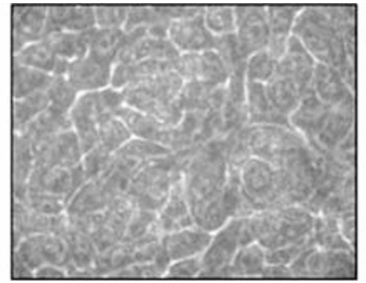

RNAi Scrambled

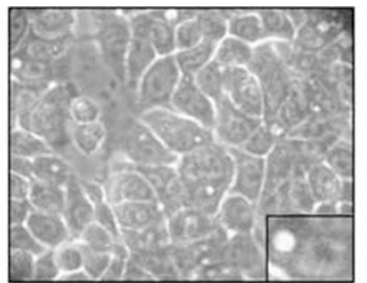

RNAi p38

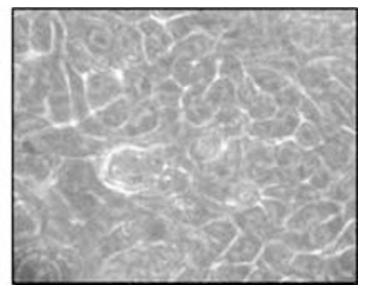

RNAi p38ß

Figure 5 SB202190-dependent induction of autophagy relies only upon p38 $\alpha$ blockade. (a, b) HT29 cells cultured in the absence or presence of NaB $1 \mathrm{mM}(48 \mathrm{~h})$ were characterized for the expression of $\mathrm{p} 38 \alpha, \beta, \gamma$ and $\delta$ isoforms. (a) Multiplex RT-PCR analysis was followed by separate PCR confirmation for each transcript. (b) Relative levels of expression of $\mathrm{p} 38 \alpha$ and $\beta$ isoforms were measured by GeneScan evaluation of fluorescent amplifications and normalized on GAPDH signals. (c-e) HT29 cells were transfected with siRNA targeting $\mathrm{p} 38 \alpha$ and $\beta$ genes. After $24 \mathrm{~h} \mathrm{RNA}$ was extracted, and the expression of $\mathrm{p} 38$ isoforms was evaluated by real-time PCR (c, upper panel). At $48 \mathrm{~h}$ post-transfection cell lysates were probed for p38 expression by immunoblot (c, lower panel), and expression of GABARAP was measured by GeneScan evaluation of fluorescent amplifications and normalized on GAPDH signals (d, lower panel). The percentage of autophagic cells was estimated by phase-contrast microscope (e) and then verified by electron microscopy evaluation (d, upper panel)

Table 1 Molecular features of the colorectal cancer cell lines used in this study

\begin{tabular}{lcccccccc}
\hline Cell line & MIN & CIN & APC & BCat & p53 & KiRas & TGF $\beta$ IIR \\
\hline Hct116 & + & - & wt & mut & wt & mut & mut \\
LS174T & + & - & wt & mut & wt & wt & mut \\
SW480 & - & + & mut & wt & mut & mut & mut \\
CaCo2 & - & + & mut & wt & mut & wt & wt \\
HT29 & - & + & mut & wt & mut & wt & wt \\
\hline
\end{tabular}

Table 2 SB202190 induces autophagic cell death of colorectal cancer cells in a cell type-specific manner

\begin{tabular}{|c|c|c|c|c|c|c|c|}
\hline Cell Line & Species & Tissue & Morphology & Origin & Tumor Type & p53 & SB-mediated autophagic cell death \\
\hline Hct116 & Human & Adult colon & Epithelial & Endodermal & Adenocarcinoma & wt & + \\
\hline CaCo2 & Human & Adult colon & Epithelial & Endodermal & Adenocarcinoma & mut & + \\
\hline SW480 & Human & Adult colon & Epithelial & Endodermal & Adenocarcinoma & mut & + \\
\hline HT29 & Human & Adult colon & Epithelial & Endodermal & Adenocarcinoma & mut & + \\
\hline LS174T & Human & Adult colon & Epithelia & Endodermal & Adenocarcinoma & wt & + \\
\hline Нер3В & Human & Child liver & Epithelial & Endodermal & Hepatocellular carcinoma & mut & - \\
\hline HeLa & Human & Fetal cervix & Epithelial & Mesodermal & Cervical carcinoma & wt & - \\
\hline $\mathrm{RD}$ & Human & Fetal muscle & Myoblast & Mesodermal & Rhabdomyosarcoma & mut & - \\
\hline HEK293 & Human & Embryonic kidney & Epithelial & Mesodermal & & wt & - \\
\hline hFSF & Human & Foetal skin & Fibroblast & Mesodermal & & wt & - \\
\hline NIH3T3 & Mouse & Embryo & Fibroblast & Mesodermal & & wt & - \\
\hline $\mathrm{CH} 310 \mathrm{~T} 1 / 2$ & Mouse & Embryo & Fibroblast & Mesodermal & & wt & - \\
\hline $\mathrm{C} 2 \mathrm{C} 12$ & Mouse & Adult muscle & Myoblast & Mesodermal & & wt & - \\
\hline
\end{tabular}



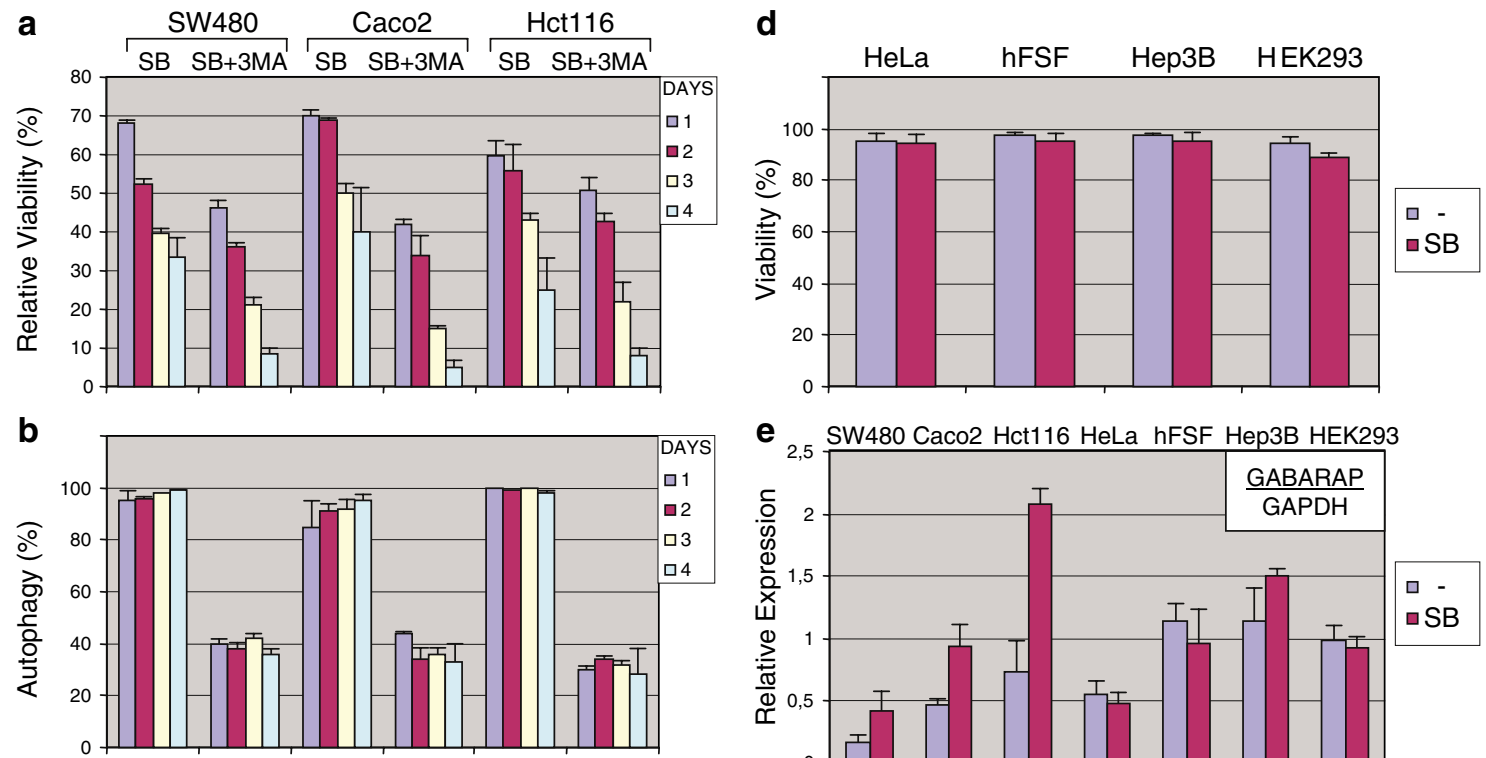

e SW480 Caco2 Hct116 HeLa hFSF Hep3B HEK293
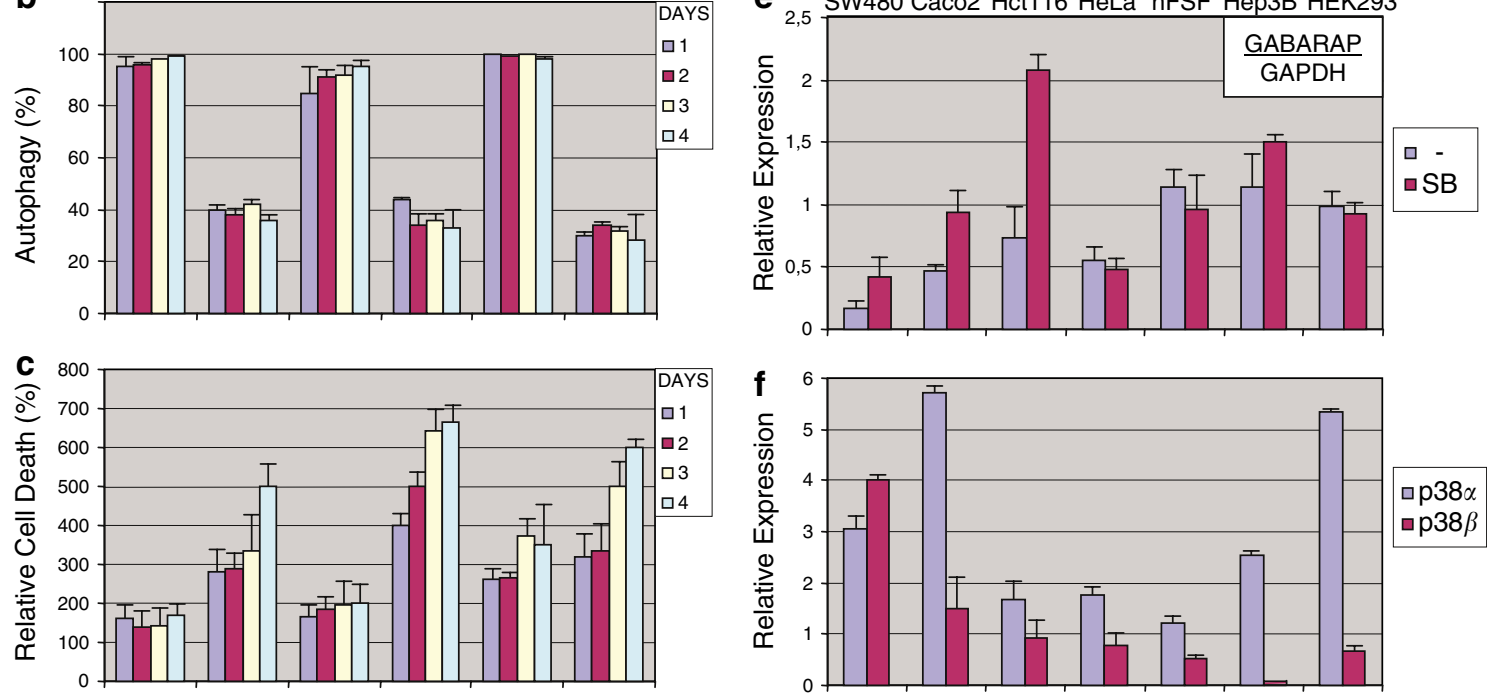

Figure 6 SB202190 induces autophagic cell death in colorectal cancer cells in a cell type-specific manner. (a-c) SW480, CaCo2 and Hct116 cells were cultured in the absence or presence of SB202190 (SB) or a combination of SB202190 and 3-methyiladenine (3MA) for the indicated period of time. Cells were suspended in a trypan blue solution or in a dye mix containing DAPI and ethidium bromide. (a) Relative viability (\%) was estimated by calculating the ratio between viable cells in treated (SB, SB $+3 \mathrm{MA}$ ) versus untreated (-) HT29. (b) The percentage of autophagic cells was estimated by phase-contrast microscopy and then verified by electron microscope evaluation. (c) Relative cell death (\%) represents the ratio between dead cells in treated versus untreated HT29. (d) Equal number of HeLa, hFSF, Hep3B and HEK293 cells were seeded and cultured in the absence or presence of SB202190 for $24 \mathrm{~h}$. Cells were scored for autophagic activity (data not shown), and viability by trypan blue staining (viable cells/total number of cells). (e, f) SW480, CaCo2, Hct116, HeLa, hFSF, Hep3B and HEK293 cells were cultured in the absence or presence of SB202190 for $24 \mathrm{~h}$. Relative abundance of GABARAP (e) or p38 $\alpha$ and $\beta$ (f) transcripts in the indicated cell lines were evaluated by GeneScan estimation of fluorescent RT-PCR products and normalization on GAPDH

Very interestingly, pretreatment with 3MA or puromycin reduces the number and size of vacuoles together with the percentage of autophagic cells in SB-treated CRCs, whereas the induction of GABARAP expression remains unaffected. $3 \mathrm{MA}$ controls the activity of the $\mathrm{Pl}_{3} \mathrm{~K}$ class III enzyme which is important in the early stages of autophagic vescicle formation by inducing the assembly of autophagosome precursors. ${ }^{22,23}$ In yeast, $\mathrm{Pl}_{3} \mathrm{~K} / \mathrm{Vps} 34$ forms a multiprotein complex including also Atg6 and Atg14. In humans, the mammalian homolog of Atg6, named Beclin-1, has been found mutated in cancer cells revealing its causative role for autophagy deficiency in cancerogenesis. ${ }^{20}$

Autophagy is a cellular process that can finally consent cell survival in stress conditions or trigger type II cell death depending on the cell type and specific stimuli. ${ }^{24,28}$ Recent work indicates that several intracellular mediators of autophagy are deregulated during malignant transformation, with a consequent reduction of the autophagic activity. ${ }^{20,24,29}$ Cancer cell lines often have lower autophagic capacity than their normal counterparts and fail to respond to serum/amino- acid deprivation or high cell density. A decrease in autophagic capacity has been also observed during animal experimental carcinogenesis. ${ }^{20}$ Another point underscoring the relevance of autophagy in cancer cells derives from the observation that some malignant cell types respond to chemotherapy or radiation by triggering autophagy, indicating the potential utility of autophagic cell death induction in cancer therapy. ${ }^{30}$ Treatment with rapamycin, temozolomide or arsenic trioxide of malignant glioma cells induces an autophagic cell death response. ${ }^{31-33}$ However that's not always the case. In fact, characterization of the effects of two DNA-damaging agents, such as temozolide and etoposide, suggests that these chemotherapy compounds induce an autophagy-associated ATP surge that protects cells contributing to drug resistance of malignant glioma.$^{34}$ Moreover, autophagy could represent a defense mechanism against sulforaphane-induced apoptosis in prostate cancer cells and against DNA-damaging agents in breast cancer. ${ }^{35,36}$ It has been recently shown that when cells with defects in apoptosis are subjected to metabolic stress, they activate autophagy to survive. Inhibition of autophagy 
triggers a necrotic pathway leading to cell death. In solid tumors, this necrosis is associated with inflammation, which creates a microenvironment favoring survival and increased proliferation of a small population of damaged cells. The final effect of this process is the enhancement of tumor growth. ${ }^{37}$ Thus, it seems that the role of autophagy in triggering survival or death in response to cancer therapies may vary depending on different cell types and treatments.

According with our results, it seems that $p 38 \alpha$ blockade in CRCs inhibits a survival pathway leading to autophagy, which probably initially represents a defense mechanism to survive. Along the treatment, these autophagic cells die with features of non-apoptotic death indicating the occurrence of autophagic cell death or type II programmed cell death. Although the increased percentage of dead cells caused by 3MA-dependent inhibition of autophagy could appear in conflict with this hypothesis, but these cells actually showed distinctive traits of apoptosis. To explain these data we propose a model suggesting that autophagy represents a survival response to $\mathrm{p} 38 \alpha$ blockade, but prolonged inactivation of the kinase leads to cell death. Inhibition of autophagy breaks this process and triggers an apoptotic response. The effect of SB treatment in CRCs recalls that of arsenic trioxide in glioma cells. In fact, the addition of an autophagy inhibitor enhances the antitumor effect through the switch between autophagic to apoptotic cell death. ${ }^{33}$ In accordance with this hypothesis, removal of SB within the first $48 \mathrm{~h}$ of treatment induces a significant time-dependent reduction in vacuole number and size together with a slow re-entry in the cell cycle with a dubling time of about $80 \mathrm{~h}$ (Comes and Simone, unpublished results).

Our experiments reveal that SB induces autophagic cell death in a cell type-specific manner as normal cells and cancer cells of different origin fail to respond to $p 38 \alpha$ blockade. Therefore, our findings establish a rationale for the evaluation of p38 $\alpha$-specific inhibition as potential therapeutic tool, alone or in combination with other compounds, in the treatment of colorectal cancer. In fact, as reported in Table 1, the $\mathrm{CRC}$ lines used in these study display significant differences both in genotype and phenotype. None of the molecular characteristics of these cells seem to be correlated with the common autophagic cell death response to SB. Moreover, $B A L B / c$ mice treated daily by intraperitoneal injection of SB for nine days survived without body and colon weight changes. It is noteworthy that the colon of these mice showed normal architecture at the histological analysis. ${ }^{38}$ Furthermore, a novel strategy for local delivery of SB has been developed to treat renal fibrosis, ${ }^{39}$ indicating that pharmacological manipulation of p38 is emerging as a potential therapeutic tool for a number of human diseases. In this sense, our study might have an even broader interest. In conclusion, the experimental model we have described can represent a useful system to study human autophagy in vitro.

\footnotetext{
Materials and Methods

Reagents. SB202190 $(10 \mu \mathrm{M}), \quad$ PD98059 $(20 \mu \mathrm{M}), \quad$ LY294002 $(25 \mu \mathrm{M})$, rapamycin $(100 \mathrm{nM})$ and $\mathrm{NaB}(1-5 \mathrm{mM})$ were purchased from Calbiochem; puromycin $(10 \mu \mathrm{M})$, 3-methyladenine (10 mM), Sudan black B, Oil red O, trypan blue, DAPI and ethidium bromide were purchased from Sigma.
}

Cell culture and microscopic quantitation of proliferation, viability and cell death. HT29, SW480, Hct116 and Caco2 cells were cultured as described. ${ }^{40}$ LS174T, Hep3B, HeLa, HEK293, C2C12, CH310T1/2, $\mathrm{NIH} 3 \mathrm{~T} 3$ and RD cells were maintained in DMEM with $10 \% \mathrm{FBS}$. hFSF were isolated and cultured as described. ${ }^{40}$ The proliferation, viability and cell death of the reported cell lines were scored by cell counting. Briefly, at the indicated time points the supernatants (containing dead/floating cells) were collected, and the remaining adherent cells were detached with Trypsin/EDTA. After that, both cell suspensions were mixed and cell pellets were obtained. Cell pellets were suspended in PBS $1 \times$ and then $10 \mu \mathrm{l}$ were mixed with equal volume of $0.01 \%$ trypan blue solution. The mixture was then transferred to the hemacytometer. Viable cells (unstained cells) and dead cells (stained cells) were counted with a phase-contrast microscope $(\times 40)$. To score the proliferation rate the entire counting grid was analyzed, whereas to evaluate viability 200 cells were counted. To determine the percentage of cells dying for apoptotic or non-apoptotic cell death, $25 \mu \mathrm{l}$ of cell suspensions were mixed with $1 \mu \mathrm{l}$ of dye mix containing DAPI $(1 \mu \mathrm{g} / \mathrm{ml})$ and ethidium bromide $(100 \mu \mathrm{g} / \mathrm{m})$. The mixture was placed on a microscope slide and covered with a $22 \mathrm{~mm}^{2}$ coverslip. The slides were examined with the 'Zeiss Axioskop 2 plus' fluorescent microscope $(\times 400)$. For each sample 200 cells were counted and recorded as V (viable cells), NVN (non-viable cells with normal nuclei) and NVA (non-viable cells with apoptotic nuclei). The $\%$ of apoptotic versus non-apoptotic dead cells was calculated (\% apoptotic cells $=100 \times \mathrm{NVA} /(\mathrm{VA}+\mathrm{NVN}+\mathrm{NVA}) ; \%$ non-apoptotic cells $=100 \times$ NVN/(VA + NVN + NVA)).

To avoid possible influence of cell density on cell growth and survival, every set of experiments (at least three replicates) was performed by seeding cells at different densities: from $50 \times 10^{3}$ to $500 \times 10^{3}$ cells in six-well dishes and from $500 \times 10^{3}$ to $1000 \times 10^{3}$ in 60 -mm-diameter dishes. The data shown in the 'Results' section are representative of three or more independent sets of experiments.

DNA fragmentation assay. The supernatants were mixed with the remaining adherent cells detached with Trypsin/EDTA, and cell pellets were suspended in hypotonic lysis buffer. After centrifugation at 14000 r.p.m. for $20 \mathrm{~min}$, proteinase $\mathrm{K}$ and then RNAsi A, was added. Samples were subjected to double phenol/chloroform extraction, DNA precipitation, suspension in TE and loaded into $1 \%$ agarose gel.

RNA interference. HT29 cells were seeded at $1500 \times 10^{3}$ cells/well in six-well dishes. Twenty-four hours after seeding, cells were transfected with $200 \mathrm{pmol}$ of siGenome SMARTpool reagent from DHARMACON (M-003512-05-0005) containing four siRNA duplexes direct against $\mathrm{p} 38 \alpha$ or $p 38 \beta$ open-reading frame using $10 \mu \mathrm{l}$ of Lipofectamine 2000 (Invitrogen, Carlsbad, CA, USA).

RNA extraction and cDNA synthesis. Total RNA was extracted with TRIzol reagent (Invitrogen, Carlsbad, CA, USA) following the manufacturer's instruction. To avoid possible DNA contaminations, RNA was treated with DNAase1 (Ambion, Austin, TX, USA). RNA purity was also checked by spectrophotometer and RNA integrity by examination on agarose gel electrophoresis. In every experiment, one to four micrograms of total RNA were retro-transcribed using High Capacity DNA Archive Kit (Applied Biosystem, Foster City, CA, USA) and following the manufacturer's instruction.

Semiquantitative RT-PCR. RT-PCR was performed using specific primers whose sequence is available on request. Standard curves were performed using serial dilutions of CDNA to define the dynamic range and calculate the amplification efficiency (see Figure 1). The PCR conditions were the following: 5 min at $94^{\circ} \mathrm{C}$ for one cycle; $30 \mathrm{~s}$ at $94^{\circ} \mathrm{C}, 30 \mathrm{~s}$ at $59-62^{\circ} \mathrm{C}$ and $1 \mathrm{~min}$ at $68^{\circ} \mathrm{C}$ for $22-28$ cycles. To quantify the relative abundance of gene-specific transcripts, fluorescent-labelled PCR products were analyzed on an ABI310 sequencer (Applied Biosystems, Foster City, CA, USA) with GeneScan software following the manufacturer's instruction, and normalized to GAPDH transcripts (see Supplementary Figure 1). Multiplex RTPCR was performed with Quiagen Multiplex PCR Master Mix using specific primers described previously. ${ }^{25}$

Quantitative real-time PCR. To detect mRNA expression levels for the p38 $\alpha$ and $\beta$ genes, primers were designed using Primer Express software spanning intron/exon boundaries whenever possible. PCR assays were performed in 96-well optical reaction plates using the ABI $7500 \mathrm{HT}$ machine (Applied Biosystem, Foster City, CA, USA). PCR assays were conducted in triplicate wells for each sample. The following reaction mixture per well was used: $10 \mu \mathrm{l}$ Power Syber Green (Applied 
Biosystem, Foster City, CA, USA), $2.4 \mu \mathrm{l}$ of primers at the final concentration of $150 \mathrm{nM}, 4.6 \mu \mathrm{l}$ RNAase free water, $3 \mu \mathrm{l}$ cDNA. For all experiments the following PCR conditions were used: $95^{\circ} \mathrm{C}$ for $10 \mathrm{~min}$, followed by 40 cycles at $95^{\circ} \mathrm{C}$ for $15 \mathrm{~s}$ then at $60^{\circ} \mathrm{C}$ for $60 \mathrm{~s}$. Quantitative normalization of CDNA in each sample were performed using cyclophillin mRNA as internal control. Relative quantification was carried out using the $\Delta \Delta C_{T}$ method. Mock-transfected cells were used as calibrator to determine relative expression level.

Immunoblot assay. Immunoblots were performed according to the Cell Signaling instructions. Briefly, cells were homogenized by sonication in $1 \times$ SDS sample buffer $(62.5 \mathrm{mM}$ Tris-HCl pH 6.8, $2 \%$ w/v SDS, $10 \%$ glycerol and $50 \mathrm{mM}$ DTT). $50 \mu \mathrm{g}$ of protein extract from each sample were electrophoresed in a $10 \%$ SDS-polyacrylamide gel and transferred to Hybond-P PVDF membrane (Amersham Biosciences, Piscataway, NJ, USA). The blots were blocked $1 \mathrm{~h}$ at RT in blocking buffer $(1 \times$ TBS, $0.1 \%$ Tween-20 with $5 \%$ w/v non-fat dry milk) and washed with TBS/T. The membranes were incubated with anti-p38 antibody (Cell Signaling; $1: 1000)$ in $10 \mathrm{ml}$ primary antibody dilution buffer $(1 \times \mathrm{TBS}, 0.1 \%$ Tween-20 with $5 \%$ BSA) overnight at $4{ }^{\circ} \mathrm{C}$. After that, the blots were extensively washed and incubated with the anti-rabbit secondary horseradish peroxidase conjugate antibody (1:20 000 Amersham Biosciences, Piscataway, NJ, USA) in blocking buffer for $45 \mathrm{~min}$, then washed and developed with the ECL chemiluminescence reagent (Amersham Biosciences, Piscataway, NJ, USA) as directed by the manufacturer.

Morphological evaluation. Numerous slides with a monolayer cells were either rapidly fixed in $95 \%$ ethyl alcohol for a minimum of $15 \mathrm{~min}$ (before staining with Papanicolaou and PAS) or unfixed (before staining for lipids with Sudan black B, Oil red 0). Slides were examined by optic microscopy (Nikon Eclipse 80i).

Measurement of vacuole size. Digital images of living vacuolated cells were imported on Adobe Photoshop software, version 7.0. The diameter of each vacuole in 50 randomly selected cells was measured and expressed in a pixel scale. The length of one grid in a micro-grid slide was also measured in a pixel scale. The diameter of each vacuole on a pixel scale was converted into micrometers based on the pixels of the standard grid length. The resolution of all the images was kept constant during the whole procedure.

Electron microscopy. Cells were fixed in $3 \%$ GTA in phosphate buffer $0.1 \mathrm{M}$ for $2 \mathrm{~h}$, washed in the same buffer and then post-fixed in $1 \% \mathrm{OsO}_{4}$ at $4{ }^{\circ} \mathrm{C}$ Afterwards, cells were scraped with a rubber bar, dehydrated in graded ethanols, and embedded in Epon 812. Ultrathin sections $(60 \mathrm{~nm})$ were cut with a diamond knife on a LKB-V ultratome, stained with uranyl acetate followed by lead cytrate and examined under a 'Zeiss 9A' electron microscope. To score autophagy 100 cells for each sample were counted.

Acknowledgements. We thank Marie Basso for her editorial assistance; Drs FP Jori, N Resta and A Stella, for their helpful discussion during the preparation of the manuscript. This work was partly supported by a grant from the Associazione Italiana Ricerca sul Cancro (to AM) and a grant from Fondazione CARIME (to GG)

1. Samoha S, Arber N. Cyclooxygenase-2 inhibition prevents colorectal cancer: from the bench to the bed side. Oncology 2005; 69 (Suppl 1): 33-37.

2. McGartland LP, Mulcahy MF, Benson III AB. Pre- and postoperative adjuvant therapy for locally advanced rectal cancer. Clin Adv Hematol Oncol 2004; 2: 806-814.

3. Medina V, Afonso JJ, Alvarez-Arquelles H, Hernandez C, Gonzalez F. Sodium butyrate inhibits carcinoma development in a 1,2-dimethylhydrazine-induced rat colon cancer J Parenter Enteral Nutr 1988; 22: 14-17.

4. Van den Brink GR, Bleuming SA, Hardwick JC, Schepman BL, Offerhaus GJ, Keller JJ et al. Indian Hedgehog is an antagonist of Wnt signaling in colonic epithelial cell differentiation. Nat Genet 2004; 36: 277-282.

5. Luongo D, Mazzarella G, Della RF, Maurano F, Rossi M. Down-regulation of ERK1 and ERK2 activity during differentiation of the intestinal cell line HT-29. Mol Cell Biochem 2002; 231: 43-50.

6. Orchel A, Dzierzewicz Z, Parfiniewicz B, Weglarz L, Wilczok T. Butyrate-induced differentiation of colon cancer cells is PKC and JNK dependent. Dig Dis Sci 2005; 50 : 490-498.

7. Archer SY, Johnson J, Kim HJ, Ma Q, Mou H, Daesety V et al. The histone deacetylase inhibitor butyrate downregulates cyclin B1 gene expression via a p21/WAF-1-dependen mechanism in human colon cancer cells. Am J Physiol Gastrointest Liver Physiol 2005; 289: G696-G703.
8. Schwab M, Reynders V, Ulrich S, Zahn N, Stein J, Schroder O. PPARgamma is a key target of butyrate-induced caspase-3 activation in the colorectal cancer cell line Caco-2. Apoptosis 2006; 11: 1801-1811.

9. Laprise P, Chailler P, Houde M, Beaulieu JF, Boucher MJ, Rivard N. Phosphatidylinositol 3-Kinase controls human intestinal epithelial cell differentiation by promoting adherens junction assembly and p38 MAPK activation. J Biol Chem 2002; 277: 8226-8234.

10. Houde M, Laprise P, Jean D, Blais M, Asselin C, Rivard N. Intestinal epithelial cell differentiation involves activation of p38 mitogen-activated protein kinase that regulates the homeobox transcription factor CDX2. J Biol Chem 2001; 276: 21885-21894.

11. Simone C, Forcales SV, Hill DA, Imbalzano AN, Latella L, Puri PL. p38 pathway targets SWI-SNF chromatin-remodeling complex to muscle-specific loci. Nat Genet 2004; 36: 738-743.

12. Simone C. SWI/SNF: the crossroads where extracellular signaling pathways meet chromatin. J Cell Physiol 2006; 207: 309-314.

13. Hiramoto T, Nakanishi T, Sumiyoshi T, Fukuda T, Matsuura S, Tauchi $\mathrm{H}$ et al. Mutations of a novel human RAD54 homologue, RAD54B, in primary cancer. Oncogene 1999; 18: 3422-3426.

14. Faust D, Dolado I, Quadrado A, Oesch F, Weiss C, Nebrada AR et al. p38alpha MAPK is required for contact inhibition. Oncogene 2005; 24: 7941-7945.

15. Surez-Cuervo C, Merrell MA, Watson L, Harris KW, Rosenthal EL, Vaananen HK et al. Breast cancer cells with inhibition of p38alpha have decreased MMP-9 activity and exhibit decreased bone metastasis in mice. Clin Exp Metastasis 2004; 21: 525-533.

16. Gout S, Morin C, Haule F, Huot J. Death receptor-3, a new E-Selectin counter-receptor that confers migration and survival advantages to colon carcinoma cells by triggering p38 and ERK MAPK activation. Cancer Res 2006; 66: 9117-9124.

17. Demidov ON, Kek C, Shreeram S, Timoffev O, Fornace AJ, Appella E et al. The role of the MKK6/p38 MAPK pathway in Wip1-dependent regulation of ErbB2-driven mammary gland tumorigenesis. Oncogene 2006; (E-pub ahead of print)

18. Bakin AV, Rinehart C, Tomlinson AK, Harteaga CL. p38 mitogen-activated protein kinase is required for TGFbeta-mediated fibroblastic transdifferentiation and cell migration. J Cell Sci 2002; 115: 3193-3206.

19. Halawani $D$, Mondeh R, Stanton LA, Beier F. p38 MAP kinase signaling is necessary for rat chondrosarcoma cell proliferation. Oncogene 2004; 23: 3726-3731.

20. Gozuacik D, Kimchi A. Autophagy as cell death and tumor suppressor mechanism. Oncogene 2004; 23: 2891-2906.

21. Abeliovich H, Dunn Jr WA, Kim J, Klionsky DJ. Dissection of autophagosome biogenesis into distinct nucleation and expansion steps. J Cell Biol 2000; 151: 1025-1034.

22. Tassa A, Roux MP, Attaix D, Bechet DM. Class III phosphoinositide 3-kinase-Beclin1 complex mediates the amino acid-dependent regulation of autophagy in $\mathrm{C} 2 \mathrm{C} 12$ myotubes. Biochem J 2003; 376: 577-586.

23. Petiot A, Ogier-Denis E, Blommaart EF, Meijer AJ, Codogno P. Distinct classes of phosphatidylinositol $3^{\prime}$-kinases are involved in signaling pathways that control macroautophagy in HT-29 cells. J Biol Chem 2000; 275: 992-998.

24. Tsujimoto Y, Shimizu S. Another way to die: autophagic programmed cell death. Cell Death Differ 2005; 12 (Suppl 2): 1528-1534.

25. Vachon PH, Harnois C, Grenier A, Dufour G, Bouchard V, Han J et al. Differentiation stateselective roles of p38 isoforms in human intestinal epithelial cell anoikis. Gastroenterology 2002; 123: 1980-1991.

26. Vom Dahl S, Dombrowski F, Schmitt M, Schliess F, Pfeifer U, Ussinger D. Cell hydration controls autophagosome formation in rat liver in a microtubule-dependent way downstream from p38MAPK activation. Biochem J 2001; 354: 31-36.

27. Prick T, Thumm M, Kohrer K, Haussinger D, Vom Dahl S. In yeast, loss of Hog1 leads to osmosensitivity of autophagy. Biochem J 2006; 394: 153-161.

28. Kabeya Y, Mizushima N, Yamamoto A, Oshitani-Okamoto S, Ohsumi Y, Yoshimori T. LC3, GABARAP and GATE16 localize to autophagosomal membrane depending on form-II formation. J Cell Sci 2004; 117: 2805-2812.

29. Codogno P, Meijer AJ. Autophagy and signaling: their role in cell survival and cell death. Cell Death Differ 2005; 12 (Suppl 2): 1509-1518

30. Marx J. Autophagy: is it cancer's friend or foe? Science 2006; 312: 1160-1161.

31. Takeuchi H, Kondo Y, Fujiwara K, Kanzawa T, Aoki H, Mills GB et al. Synergistic augmentation of rapamycin-induced autophagy in malignant glioma cells by phosphatidylinositol 3-kinase/protein kinase B inhibitors. Cancer Res 2005; 65: 3336-3346.

32. Kanzawa $\mathrm{T}$, Germano IM, Komata $\mathrm{T}$, Ito $\mathrm{H}$, Kondo $\mathrm{Y}$, Kondo $\mathrm{S}$. Role of autophagy in temozolomide-induced cytotoxicity for malignant glioma cells. Cell Death Differ 2004; 11 : 448-457.

33. Kanzawa $\mathrm{T}$, Kondo $\mathrm{Y}$, Ito $\mathrm{H}$, Kondo $\mathrm{S}$, Germano I. Induction of autophagic cell death in malignant glioma cells by arsenic trioxide. Cancer Res 2003; 63: 2103-2108.

34. Katayama M, Kawaguchi T, Berger MS, Pieper RO. DNA damaging agent-induced autophagy produces a cytoprotective adenosine triphosphate surge in malignant glioma cells. Cell Death Differ 2006; 1 September; (E-pub ahead of print).

35. Herman-Antosiewicz A, Johnson DE, Singh SV. Sulforaphane causes autophagy to inhibit release of cytochrome $c$ and apoptosis in human prostate cancer cells. Cancer Res 2006; 66: 5828-5835.

36. Abedin MJ, Wang D, McDonnell MA, Lehmann U, Kelekar A. Autophagy delays apoptotic death in breast cancer cells following DNA damage. Cell Death Differ 2006; 22 September; (E-pub ahead of print). 
37. Degenhardt K, Mathew R, Beaudoin B, Bray K, Anderson D, Chen G et al. Autophagy promotes tumor cell survival and restricts necrosis, inflammation, and tumorigenesis. Cancer Cell 2006; 10: 51-64.

38. Ten Hove T, van den Blink B, Pronk I, Drillenburg P, Peppelenbosch MP, van Deventer SJ. Dichotomal role of inhibition of p38 MAPK with SB 203580 in experimental colitis. Gut 2002; 50: 507-512.
39. Prakash J, Sandovici M, Saluja V, Lacombe M, Schaapveld RQ, de Borst $M$ et al. Intracellular delivery of the P38 MAPK inhibitor SB202190 in renal tubular cells: a nove strategy to treat renal fibrosis. J Pharmacol Exp Ther 2006; 319: 8-19.

40. Corin I, Di Giacomo MC, Lastella P, Bagnulo R, Guanti G, Simone C. Tumor-specific hyperactive low-molecular-weight cyclin $\mathrm{E}$ isoforms detection and characterization in nonmetastatic colorectal tumors. Cancer Biol Ther 2006; 5: 198-203.

Supplementary Information accompanies the paper on Cell Death and Differentiation website (http://www.nature.com/cdd) 